\title{
Electromagnetic activity: a possible player in epilepsy
}

\author{
Yi Guo, Yaowen Liu and Xuefeng Wang*
}

\begin{abstract}
Epilepsy is a common disease with frequent occurrences. Many precipitating factors contribute to epileptic seizures, such as hyperventilation and alcohol consumption. An increasing number of studies have also found that electromagnetic activity in the environment can also affect epileptic seizures. However, many neuromodulatory devices that produce electromagnetic fields have been applied in the diagnosis and treatment of epilepsy. In this paper, we performed literature search in the PubMed, Medline and EMBASE databases and reviewed retrospective, prospective, or cross-sectional studies and case reports on the effects of electromagnetic activity on epilepsy. The application of electromagnetic activity in the diagnosis and treatment of epilepsy is also reviewed.
\end{abstract}

Keywords: Electromagnetic activity, Epilepsy, Electroencephalogram, Magnetoencephalography, Repetitive transcranial magnetic stimulation

\section{Introduction}

Epilepsy is a common neurological disease characterized by recurrent, transient, rigid, and usually self-limiting seizures, with a global prevalence of $7 \%$ according to the epidemic surveying data, representing approximately 50 million epilepsy patients worldwide [1,2]. Epilepsy can affect people of all ages, and nearly $50 \%$ of patients with newly-diagnosed epilepsy do not become seizure-free after antiepileptic drug (AED) treatment [3]. In addition, various factors may trigger seizures in patients with epilepsy, such as alcohol consumption and hyperventilation $[4,5]$. Numerous studies have revealed that electromagnetic activity in the environment can also trigger seizures in epileptic patients [6, 7]. However, many neuronal modulatory devices that produce electromagnetic fields have been introduced to diagnose and treat epilepsy [8-10]. In this paper, we summarize the effects of electromagnetic activity on epilepsy and the applications of electromagnetic activity in the diagnosis and treatment of epilepsy by systematically reviewing literature searched in the PubMed, Medline and EMBASE databases.

\footnotetext{
* Correspondence: xfyp@163.com

Department of Neurology, The First Affiliated Hospital of Chongqing Medical University, Chongqing Key Laboratory of Neurology, Chongqing 400016, China
}

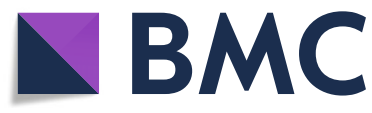

(C) The Author(s). 2020 Open Access This article is licensed under a Creative Commons Attribution 4.0 International License, which permits use, sharing, adaptation, distribution and reproduction in any medium or format, as long as you give

appropriate credit to the original author(s) and the source, provide a link to the Creative Commons licence, and indicate if changes were made. The images or other third party material in this article are included in the article's Creative Commons licence, unless indicated otherwise in a credit line to the material. If material is not included in the article's Creative Commons licence and your intended use is not permitted by statutory regulation or exceeds the permitted use, you will need to obtain permission directly from the copyright holder. To view a copy of this licence, visit http://creativecommons.org/licenses/by/4.0/

\section{Sources of electromagnetic waves and fields}

The living environment of mankind itself is a large magnetic field; the thermal radiation from the earth's surface and lightening are both able to produce electromagnetic waves, and the sun and other stars continuously transmit electromagnetic radiation to the earth from outer space; these help to form the earth's natural electromagnetic fields. With the progress of science and technology, the application of electromagnetic technology has contributed enormously to the progress of human society and civilization, while at the same time producing large amounts of electromagnetic radiation waves in our daily life. The electromagnetic waves, which are created as a result of vibrations between an electric field and a magnetic field, has since the 1970s been considered as the fourth largest source of pollution by the World Health Organization, only next to the air pollution, water pollution and noise pollution $[11,12]$.

\section{Epileptogenic effect of electromagnetic activities Electromagnetic waves aggravate epileptic seizures Electromagnetic activity has been demonstrated to in- crease the susceptibility to epilepsy in animal studies. Michon et al. [13] exposed chronic epileptic rats to arti- ficial magnetic fields that simulated the magnitude and}


morphology of enhanced geomagnetic activity for $5 \mathrm{~min}$ once per hour from midnight to 8:00 a.m. in next morning with changing intensities (from $0 \mathrm{nT}$ to $70 \mathrm{nT}$ in increments of 15 or $20 \mathrm{nT}$ for $30 \mathrm{~s}$ ) and revealed that the exposed rats had increased occurrence of seizures the next day. Exposure to increased magnitudes of daily natural geomagnetic activity (regional range approximately 10-70 $\mathrm{nT}$ ) or nocturnal exposure to experimental magnetic fields that simulated geomagnetic activity with incremental changes in intensity over time significantly elevated the incidence of seizures in epileptic rats during the observational period $[14,15]$. In rats with lithium and pilocarpine injections, Bureau et al. [16] found that when the intensity of artificial, enhanced geomagnetic activity exceeded $20-25 \mathrm{nT}$, the seizures occurred more quickly than that under average daily geomagnetic activity. However, whether the effects of electromagnetic activity occur in epileptic patients remain unknown.

\section{Electromagnetic waves evoke seizure activity}

Repetitive transcranial magnetic stimulation (rTMS) is a noninvasive neuro-electrophysiological technique developed to treat depression, schizophrenia, Parkinson's disease, epilepsy and other psychiatric and neurological diseases [17]. However, a number of studies have reported that rTMS can induce episodic epileptic seizures in healthy individuals, nonepileptic patients, or patients with epilepsy $[18,19]$. Three of 9 healthy participants who received rapid-rate transcranial magnetic stimulation (TMS), suffered from secondary generalized seizures when stimulated at the highest intensity despite the lack of definite risk factors [20]; and two case reports each reported the development of seizure during a rTMS session in a patient with depression [19, 21]. Gómez et al. [22] reported that a 58-year-old stroke patient who attempted to use rTMS to rehabilitate left hemiparesis, developed a convulsive seizure during his first session of subthreshold. Dhuna et al. [23] found that one of 8 epilepsy patients receiving TMS developed a focal epileptic seizure induced by TMS.

\section{Electromagnetic waves are associated with sudden unexpected death in epilepsy (SUDEP)}

SUDEP is not an unusual phenomenon among patients with epilepsy. In one study, rats were first exposed to $500,50,10-40 \mathrm{nT}$ or sham (less than $10 \mathrm{nT}$ ) experimental magnetic fields for 6 min each hour from midnight to 8:00 am for three successive nights and then injected with pilocarpine to induce epileptic seizures. The rats suffered a mortality rate of $60 \%$ within $24 \mathrm{~h}$ following the injection of lithium and pilocarpine, in contrast to the mortality of $10 \%$ in the control group [24]. These results indicate that a variety of geomagnetic activity markedly elevates the mortality of kindled rats and that the electromagnetic stimulation is related to SUDEP in epilepsy. Previous studies found that most SUDEP occurred in young patients or in bedrooms, so we hypothesize that an abrupt global geomagnetic field change at night may be associated with the increased occurrence of SUDEP in epilepsy. Another study found that during a 2-year observational period in chronic epileptic rats, the incidence of SUDEP was significantly higher in rats exposed to an average daily geomagnetic activity exceeding $50 \mathrm{nT}$ that suddenly began at night [25].

\section{Relationships between electromagnetic waves and electroencephalograms (EEG)}

In 1924, German psychiatrist Berger successfully recorded human brain waves for the first time, giving rise to the term "human EEG". A changing electric field will produce a magnetic field, and changing magnetic fields are a major source of electric fields. The changing electric and magnetic fields form an inseparable unit called the electromagnetic field. The spread of electromagnetic fields in space forms electromagnetic waves. Cohen et al. [26] first detected weak alternating magnetic fields outside the human scalp produced by alpha-rhythm currents, which were magnetic fields produced by changing neural currents, and thus the human brain waves were considered as electromagnetic waves.

The human brain is a key and significant source of electromagnetic signals, supported by biochemical systems for rapid intelligence and reactions. The rhythm frequency range of EEG is consistent with the frequency range of the Schumann resonance signal $(0-45 \mathrm{~Hz})$. The Schumann resonance signal provides a brain frequency range matching the electromagnetic signal, providing a persistent synchronization system needed for stabilizing the brain [27]. During the neonatal or infancy period, the human brain waves are predominated by slow waves, and their frequency gradually increases with growth and developmental processing until adulthood. The main rhythm frequency of the background brain activity can reach $8-13 \mathrm{~Hz}$, which coincides with the main electromagnetic waves of the external environment (i.e., the Schumann resonance); therefore, the formation of the background brain activity might have been affected by the external electromagnetic activity, which is a result of the adaption of the human brain to the environment in the course of evolution [27].

Electromagnetic activity can also induce changes in background EEG activity of the brain. A study in 10 healthy subjects showed that prolonged exposure to electromagnetic stimulation by a mobile phone signal (Global System for Mobile Communication, GSM) enhanced interhemispheric temporal and frontal coherence in the alpha 2 (between 8 and $10 \mathrm{~Hz}$ ) and alpha 3 
(between 10 and $12 \mathrm{~Hz}$ ) bands, which demonstrated that the bilateral cerebral hemispheres have functional coupling but also that GSM electromagnetic waves from a mobile phone can influence cortical excitability and the spread of neural synchronization activity [28]. In addition, the elderly population is more sensitive to the GSM electromagnetic waves from a mobile phone than younger people, with a statistically significant increment of the interhemispheric coherence of the temporal and frontal alpha rhythms (between 8 and $12 \mathrm{~Hz}$ ); this has significant implications for the elderly, as epidemiologically they are at a high risk of epilepsy [29].

Epileptic patients, after exposure to the GSM electromagnetic fields of mobile phones, show a significantly higher interhemispheric coherence of frontal and temporal alpha rhythms (including alpha 2 and alpha 3) compared with the age-matched healthy control volunteers, which indicated that individuals with epilepsy are even more sensitive to mobile phone GSM electromagnetic waves and tend to be more likely to experience highly-synchronous activity in their neurons. Therefore, in the diseased state, the electromagnetic signaling system of the brain becomes unstable and more easily disrupted by epileptogenic factors, eventually leading to epilepsy [30]. High-frequency oscillations or high-rate fast ripples can be detected intermittently in the epileptogenic focus or seizure-onset zone (SOZ) by EEG in epilepsy patients, and more complete resection of this focus or the SOZ may achieve a better seizure prognosis than resection of only the SOZ, suggesting that higherfrequency brain waves have a close association with epilepsy [31, 32]. Electromagnetic waves might evoke highly synchronous and abnormal discharges among neurons, and low-frequency $(<1 \mathrm{~Hz})$ electromagnetic activity is able to induce oscillations in neocortical neurons, eliciting slow sleep oscillations through the diffuse hyperpolarization of cortical and thalamic neurons, which gradually gives rise to spike-wave seizures [33-36].

\section{Application of electromagnetic fields in the diagnosis and treatment of epilepsy Magnetoencephalography (MEG)}

MEG is a noninvasive neuroimaging technique that is widely used in the whole-head detection of magnetic fields generated by neuronal activity within the brain. In 1972, Cohen et al. detected for the first time a magnetic field and an EEG activity simultaneously in epilepsy patients and demonstrated that MEG might reveal new information in addition to regular EEG recording [37, 38]. Due to its prominent temporal and spatial resolution, MEG has been widely used for clinical diagnosis of epilepsy and pre-surgical localization of epileptogenic zones [39]. In 1990, Stefan et al. [40] performed simultaneous magnetoencephalographic electroencephalographic recordings from 8 epilepsy patients, and their results showed that MEG can provide more detailed localization information before surgery. Wheless et al. [41] conducted a prospective study to determine and compare the efficacy and relative contributions of EEG (scalp and intracranial), magnetic resonance imaging and MEG in identifying epileptogenic zones for resection in 58 patients with drugresistant focal epilepsy, and the results showed that MEG was comparable with intracranial EEG in predicting epileptogenic foci in temporal lobe epilepsy (57\% vs $62 \%)$. After that, an increasing number of studies have focused on the role of MEG in predicting the epileptogenic zone and have concluded that MEG is an excellent modality in the presurgical evaluation of patients with refractory focal epilepsy [42-45].

\section{rTMS}

In 1990s, Anninos et al. [46] reported that the external artificial magnetic field of low intensity effectively attenuated seizure frequency during an observation period of 10 to 14 months, which may be mediated by modulations of the activity of pineal gland, a magnetosensitive organ responsible for secretion of melatonin. These findings suggest that the artificial magnetic fields can be used as an adjunctive treatment for some drug-resistant epilepsy. Now the rTMS technique has become a potential neuromodulatory treatment for seizure control. An increasing number of studies has demonstrated that the application of rTMS treatment can reduce the frequency of seizures and/or epileptic discharge in drug-resistant epilepsy (Table 1), while the standard protocol remains elusive. Menkes et al. [56] reported that a patient with drug-refractory epilepsy experiences an over $2 / 3$ reduction in the frequency of seizures and interictal spikes $(70$ and $77 \%$, respectively) after low-frequency rTMS for four consecutive weeks. Shon et al. [47] also conducted a prospective study in four patients with drug-resistant epilepsy and concluded that rTMS can be used as a novel adjunctive therapy for medically-intractable epilepsy. In another study, Kinoshita et al. [54] evaluated the therapeutic effects of rTMS in adult patients with refractory extratemporal lobe epilepsy, and found that both complex partial seizures and partial seizures were significantly decreased (by 35.9 and $7.4 \%$, respectively) following 1 week of low-frequency rTMS treatment. In a randomized controlled, double-blind study employing patients with malformations of cortical development and refractory epilepsy, low-frequency rTMS significantly reduced the number of clinical epileptic seizures compared to the sham rTMS, and decreased epileptiform discharges immediately after and at week 4 session of rTMS [53]. Regarding the intensity of stimulation, in 
Table 1 Therapeutic effects of rTMS on drug-resistant epilepsy

\begin{tabular}{|c|c|c|c|c|c|}
\hline References & $\begin{array}{l}\text { Sample } \\
\text { size }\end{array}$ & Study design & Diagnosis & Therapy & Seizure control \\
\hline $\begin{array}{l}\text { Shon et al. } \\
\text { [47] }\end{array}$ & 4 & $\begin{array}{l}\text { Prospective } \\
\text { open-label trial }\end{array}$ & $\begin{array}{l}\text { Multidrug-resistant } \\
\text { focal epilepsy }\end{array}$ & $\begin{array}{l}\text { Ten-day stimulation at a daily } \\
\text { dose of } 900 \text { pulses of } 0.5 \mathrm{~Hz}\end{array}$ & Seizure reduction was observed in 3 patients \\
\hline $\begin{array}{l}\text { Gersner } \\
\text { et al.[48] }\end{array}$ & 1 & Case report & $\begin{array}{l}\text { Drug-resistant } \\
\text { temporal lobe epilepsy }\end{array}$ & $\begin{array}{l}\text { Three courses of stimulation } \\
\text { with daily dose of } 1800 \text { pulses } \\
\text { of } 1 \mathrm{~Hz}\end{array}$ & Seizure frequency decreased by $50-70 \%$ \\
\hline $\begin{array}{l}\text { Sun et al. } \\
\text { [49] }\end{array}$ & 60 & $\begin{array}{l}\text { Randomized, } \\
\text { single-blind, } \\
\text { controlled study }\end{array}$ & $\begin{array}{l}\text { Refractory focal } \\
\text { epilepsy }\end{array}$ & $\begin{array}{l}\text { Two weeks of high-intensity } \\
\text { ( } 90 \% \text { RMT) rTMS treatment }\end{array}$ & $\begin{array}{l}\text { Seizures frequency and interictal } \\
\text { dischargedecreased significantly compared with } \\
\text { baseline }\end{array}$ \\
\hline $\begin{array}{l}\text { Sun et al. } \\
\text { [50] }\end{array}$ & 17 & Open-label trial & $\begin{array}{l}\text { Not obtaining seizure- } \\
\text { free after resection of } \\
\text { epileptogenic region }\end{array}$ & $\begin{array}{l}\text { Two weeks of treatment at a } \\
\text { dose of } 500 \text { pulses of } 0.5 \mathrm{~Hz} \\
\text { and } 90 \% \text { RMT }\end{array}$ & Seizure frequency decreased by $50 \%$ \\
\hline $\begin{array}{l}\text { Santiago } \\
\text { Rodriguez } \\
\text { et al. [51] }\end{array}$ & 12 & Open-label trial & $\begin{array}{l}\text { Focal neocortical } \\
\text { epilepsy }\end{array}$ & $\begin{array}{l}\text { Two weeks of rTMS with a } \\
\text { dose of } 900 \text { pulses of } 0.5 \mathrm{~Hz} \\
\text { (with intensity of } 120 \% \mathrm{RMT} \text { ) }\end{array}$ & $\begin{array}{l}\text { Seizure frequency and interictal spikes decreased } \\
\text { significantly }\end{array}$ \\
\hline $\begin{array}{l}\text { Joo et al. } \\
\text { [52] }\end{array}$ & 35 & Not mentioned & Focal seizure & $\begin{array}{l}\text { Five consecutive rTMS with } \\
\text { an intensity of 100\% RMT }\end{array}$ & $\begin{array}{l}\text { Interictal epileptogenic discharges decreased } \\
\text { significantly while seizure frequency did not }\end{array}$ \\
\hline $\begin{array}{l}\text { Fregni } \\
\text { et al. [53] }\end{array}$ & 21 & $\begin{array}{l}\text { Randomized, } \\
\text { double-blind } \\
\text { sham-controlled } \\
\text { trial }\end{array}$ & $\begin{array}{l}\text { Drug refractory } \\
\text { epilepsy }\end{array}$ & $\begin{array}{l}\text { Five consecutive treatment } \\
\text { sessions of } 1200 \text { pulses of } 1 \\
\mathrm{~Hz}\end{array}$ & $\begin{array}{l}\text { Seizure frequency and epileptiform discharges } \\
\text { reduced significantly }\end{array}$ \\
\hline $\begin{array}{l}\text { Kinoshita } \\
\text { et al. [54] }\end{array}$ & 7 & Not mentioned & $\begin{array}{l}\text { Medically intractable } \\
\text { extratemporal lobe } \\
\text { epilepsy }\end{array}$ & $\begin{array}{l}\text { A week of low-frequency } \\
\text { rTMS at } 0.9 \mathrm{~Hz} \text { (with intensity } \\
\text { of } 90 \% \text { RMT) }\end{array}$ & $\begin{array}{l}\text { The frequency of all seizure types, complex partial } \\
\text { seizures and simple partial seizures decreased } \\
\text { significantly (19.1, 35.9 and } 7.4 \% \text {, respectively) }\end{array}$ \\
\hline $\begin{array}{l}\text { Fregni } \\
\text { et al. [55] }\end{array}$ & 8 & Not mentioned & $\begin{array}{l}\text { Refractory epilepsy and } \\
\text { malformations of } \\
\text { cortical development }\end{array}$ & $\begin{array}{l}\text { One session of low-frequency } \\
\text { rTMS at a daily dose of } 600 \\
\text { pulses of } 0.5 \mathrm{~Hz}\end{array}$ & $\begin{array}{l}\text { Seizure frequency and epileptiform discharges } \\
\text { decreased by } 50 \%\end{array}$ \\
\hline
\end{tabular}

another randomized controlled study recruiting 64 patients with refractory partial epilepsy, Sun et al. [49] demonstrated that a 90\% resting motor threshold (RMT) of low-frequency rTMS targeting the epileptogenic zone produced therapeutic effects, while lower intensity (20\% RMT) of rTMS did not show any therapeutic effect. In 2017, a meta-analysis revealed that the mean event rate of $50 \%$ seizure-reduction under the low-frequency rTMS treatment was $30 \%$, and sensitivity analysis found that an age younger than 21 years and the use of a figure- 8 coil were predictors of favorable seizure control in patients with temporal lobe epilepsy [57]. Taken together, these results showed that low-frequency rMTS may be an adjunctive option for the treatment of refractory epilepsy. However, a well-designed clinical study is needed to further confirm this issue.

As to which one is the most important parameter of rMTS in treating epilepsy, different hypotheses have arisen from different studies. The RMT (which is defined as the lowest stimulus intensity capable of evoking motor potentials of at least $50 \mu \mathrm{V}$ in a target muscle) is key to individualized therapy strategies. The frequency of stimulation was less than $1 \mathrm{~Hz}$ in the majority of studies, and the period of treatment always lasted for 1-2 weeks. The mechanism of rTMS remains elusive, however; current studies suggest that rTMS suppresses epileptic seizures by altering synaptic transmission [58], neuronal excitability and ion channel function [59], or interrupting ephaptic interactions which are critical for neuronal synchronization [60], progenitor cell proliferation and microglial activation [61].

\section{Summary}

Diseases result from disturbances of the harmonious relations between an individual and the environment, and electromagnetic activity plays a role in epilepsy. Further studies on the electromagnetic activity will advance our understanding of the role electric activity plays in epilepsy. The following issues need to be addressed in future.

First, whether electromagnetic activity can change epilepsy or seizure threshold should be clarified. To date, only a small number of case reports and animal studies have reported findings on this topic; more well-designed animal studies and large-sample clinical epidemiological studies are needed. Second, although the beneficial effects of low-frequency rTMS in reducing epileptiform discharges and seizure frequency have demonstrated potentials of this tool to be used for epilepsy treatment, due to the variability in rTMS stimulation protocols, still 
more well-designed randomized controlled trials (with a standard protocol, adequate sample size and duration) are needed to validate the long-term efficiency and safety of this therapeutic approach. Third, to better understand and employ electromagnetic activity, further basic research should focus on the neuronal network, as well as cellular and molecular mechanisms underlying the effects of electromagnetic fields, which will provide insights into new treatments for epileptic seizures.

\section{Abbreviations}

AEDs: Antiepileptic drugs; EEG: Electroencephalogram; GSM: Global System for Mobile Communication; MEG: Magnetoencephalography; RMT: Resting motor threshold; rTMS: Repetitive transcranial magnetic stimulation; SUDEP: Sudden unexpected death in epilepsy; SOZ: Seizure-onset zone

\section{Acknowledgements}

Not applicable.

\section{Authors' contributions}

Yi Guo and Yaowen Liu conceived the article and wrote the manuscript Xuefeng Wang reviewed and edited the manuscript. All authors read and approved the final version of the manuscript.

\section{Authors' information}

All authors came from the First Affiliated Hospital of Chongqing Medical University, and Pro. Xuefeng Wang is the vice president of China Association Against Epilepsy (CAAE)

\section{Funding}

This work was supported by the National Natural Science Foundation of China (No. 81671301)

\section{Availability of data and materials}

Not applicable.

\section{Ethics approval and consent to participate}

Not applicable.

\section{Consent for publication}

Not applicable.

\section{Competing interests}

All authors declared that they had no conflict of interest.

Received: 24 November 2019 Accepted: 3 June 2020

Published online: 29 June 2020

\section{References}

1. Aaberg KM, Gunnes N, Bakken IJ, Lund Soraas C, Berntsen A, Magnus P, et al. Incidence and Prevalence of Childhood Epilepsy: A Nationwide Cohort Study. Pediatrics. 2017;139(5):e20163908.

2. Fiest KM, Sauro KM, Wiebe S, Patten SB, Kwon CS, Dykeman J, et al. Prevalence and incidence of epilepsy: A systematic review and metaanalysis of international studies. Neurology. 2017;88(3):296-303.

3. Shorvon SD, Goodridge DM. Longitudinal cohort studies of the prognosis of epilepsy: contribution of the National General Practice Study of epilepsy and other studies. Brain. 2013:136(Pt 11):3497-510.

4. Salvati KA, Beenhakker MP. Out of thin air: hyperventilation-triggered seizures. Brain Res. 2019;1703:41-52

5. Hamerle M, Ghaeni L, Kowski A, Weissinger F, Holtkamp M. Alcohol use and alcohol-related seizures in patients with epilepsy. Front Neurol. 2018;9:401.

6. Ghazizadeh $\vee$, Naziroglu M. Electromagnetic radiation (Wi-fi) and epilepsy induce calcium entry and apoptosis through activation of TRPV1 channel in hippocampus and dorsal root ganglion of rats. Metab Brain Dis. 2014;29(3): 787-99

7. Cinar N, Sahin S, Erdinc OO. What is the impact of electromagnetic waves on epileptic seizures? Med Sci Monit Basic Res. 2013;19:141-5.
8. Duez L, Tankisi H, Hansen PO, Sidenius P, Sabers A, Pinborg LH, et al. Electromagnetic source imaging in presurgical workup of patients with epilepsy: a prospective study. Neurology. 2019;92(6):e576-86.

9. Islam M, Westin K, Carvalho A, Eriksson M, Lundvall M, Stödberg T, et al. MEG and navigated TMS jointly enable spatially accurate application of TMS therapy at the epileptic focus in pharmacoresistant epilepsy. Brain Stimul. 2019;12(5):1312-4.

10. Kimiskidis VK, Tsimpiris A, Ryvlin P, Kalviainen $R$, Koutroumanidis $M$, Valentin A, et al. TMS combined with EEG in genetic generalized epilepsy: a phase II diagnostic accuracy study. Clin Neurophysiol. 2017;128(2):367-81.

11. Kowall B, Breckenkamp J, Heyer K, Berg-Beckhoff G. German wide cross sectional survey on health impacts of electromagnetic fields in the view of general practitioners. Int J Public Health. 2010:55(5):507-12.

12. Leitgeb N. Comparative health risk assessment of electromagnetic fields. Wien Med Wochenschr (1946). 2011:161(9-10):251-62.

13. Michon A, Koren SA, Persinger MA. Attempts to simulate the association between geomagnetic activity and spontaneous seizures in rats using experimentally generated magnetic fields. Percept Mot Skills. 1996;82(2): 619-26.

14. Michon $\mathrm{AL}$, Persinger MA. Experimental simulation of the effects of increased geomagnetic activity upon nocturnal seizures in epileptic rats. Neurosci Lett. 1997:224(1):53-6.

15. Persinger MA. Enhancement of limbic seizures by nocturnal application of experimental magnetic fields that simulate the magnitude and morphology of increases in geomagnetic activity. Int J Neurosci. 1996;86(3-4):271-80.

16. Bureau YR, Persinger MA. Decreased latencies for limbic seizures induced in rats by lithium-pilocarpine occur when daily average geomagnetic activity exceeds 20 nanoTesla. Neurosci Lett. 1995:192(2):142-4.

17. Vidal-Dourado M, Conforto AB, Caboclo LO, Scaff M, Guilhoto LM, Yacubian EM. Magnetic fields in noninvasive brain stimulation. Neuroscientist. 2014; 20(2):112-21.

18. Pascual-Leone A, Valls-Sole J, Brasil-Neto JP, Cohen LG, Hallett M. Seizure induction and transcranial magnetic stimulation. Lancet (London, England). 1992:339(8799):997.

19. Hu SH, Wang SS, Zhang MM, Wang JW, Hu JB, Huang ML, et al. Repetitive transcranial magnetic stimulation-induced seizure of a patient with adolescent-onset depression: a case report and literature review. J Int Med Res. 2011:39(5):2039-44.

20. Pascual-Leone A, Houser CM, Reese K, Shotland LI, Grafman J, Sato S, et al. Safety of rapid-rate transcranial magnetic stimulation in normal volunteers. Electroencephalogr Clin Neurophysiol. 1993;89(2):120-30.

21. Bagati D, Mittal S, Praharaj SK, Sarcar M, Kakra M, Kumar P. Repetitive transcranial magnetic stimulation safely administered after seizure. J ECT. 2012;28(1):60-1.

22. Gomez L, Morales L, Trapaga O, Zaldivar M, Sanchez A, Padilla E, et al. Seizure induced by sub-threshold $10-\mathrm{Hz}$ rTMS in a patient with multiple risk factors. Clin Neurophysiol. 2011:122(5):1057-8.

23. Dhuna A, Gates J, Pascual-Leone A. Transcranial magnetic stimulation in patients with epilepsy. Neurology. 1991:41(7):1067-71.

24. Persinger MA, McKay BE, O'Donovan CA, Koren SA. Sudden death in epileptic rats exposed to nocturnal magnetic fields that simulate the shape and the intensity of sudden changes in geomagnetic activity: an experiment in response to Schnabel, Beblo and may. Int J Biometeorol. 2005;49(4):256-61.

25. Persinger MA, Psych C. Sudden unexpected death in epileptics following sudden, intense, increases in geomagnetic activity: prevalence of effect and potential mechanisms. Int J Biometeorol. 1995:38(4):180-7.

26. Cohen D. Magnetoencephalography: evidence of magnetic fields produced by alpha-rhythm currents. Science. 1968;161(3843):784-6.

27. Cherry NJ. Human intelligence: the brain, an electromagnetic system synchronised by the Schumann resonance signal. Med Hypotheses. 2003; 60(6):843-4.

28. Vecchio F, Babiloni C, Ferreri F, Curcio G, Fini R, Del Percio C, et al. Mobile phone emission modulates interhemispheric functional coupling of EEG alpha rhythms. Eur J Neurosci. 2007;25(6):1908-13.

29. Vecchio F, Babiloni C, Ferreri F, Buffo P, Cibelli G, Curcio G, et al. Mobile phone emission modulates inter-hemispheric functional coupling of EEG alpha rhythms in elderly compared to young subjects. Clin Neurophysiol. 2010;121(2):163-71.

30. Vecchio F, Tombini M, Buffo P, Assenza G, Pellegrino G, Benvenga A, et al. Mobile phone emission increases inter-hemispheric functional coupling of 
electroencephalographic alpha rhythms in epileptic patients. Int J Psychophysiol. 2012;84(2):164-71.

31. Akiyama T, McCoy B, Go CY, Ochi A, Elliott IM, Akiyama M, et al. Focal resection of fast ripples on extraoperative intracranial EEG improves seizure outcome in pediatric epilepsy. Epilepsia. 2011;52(10):1802-11.

32. Salami P, Levesque M, Gotman J, Avoli M. A comparison between automated detection methods of high-frequency oscillations $(80-500 \mathrm{~Hz})$ during seizures. J Neurosci Methods. 2012;211(2):265-71.

33. Steriade M, Contreras D, Amzica F, Timofeev I. Synchronization of fast (30-40 $\mathrm{Hz}$ ) spontaneous oscillations in intrathalamic and thalamocortical networks. J Neurosci. 1996;16(8):2788-808.

34. Steriade M, Amzica F. Coalescence of sleep rhythms and their chronology in corticothalamic networks. Sleep Res Online. 1998;1 (1):1-10.

35. Steriade M, Nunez A, Amzica F. A novel slow $(<1 \mathrm{~Hz})$ oscillation of neocortical neurons in vivo: depolarizing and hyperpolarizing components. J Neurosci. 1993;13(8):3252-65.

36. Steriade M, Amzica F, Neckelmann D, Timofeev I. Spike-wave complexes and fast components of cortically generated seizures. II. Extra- and intracellular patterns. J Neurophysiol. 1998;80(3):1456-79.

37. Hamandi K, Routley BC, Koelewijn L, Singh KD. Non-invasive brain mapping in epilepsy: applications from magnetoencephalography. J Neurosci Methods. 2016;260:283-91.

38. Cohen D. Magnetoencephalography: detection of the brain's electrical activity with a superconducting magnetometer. Science. 1972;175(4022):664-6.

39. Schwartz ES, Edgar JC, Gaetz WC, Roberts TP. Magnetoencephalography. Pediatr Radiol. 2010;40(1):50-8.

40. Stefan H, Schneider S, Abraham-Fuchs K, Bauer J, Feistel H, Pawlik G, et al. Magnetic source localization in focal epilepsy. Multichannel magnetoencephalography correlated with magnetic resonance brain imaging. Brain. 1990;113(Pt 5):1347-59.

41. Wheless JW, Willmore LJ, Breier Jl, Kataki M, Smith JR, King DW, et al. A comparison of magnetoencephalography, MRI, and V-EEG in patients evaluated for epilepsy surgery. Epilepsia. 1999;40(7):931-41.

42. Englot DJ, Nagarajan SS, Imber BS, Raygor KP, Honma SM, Mizuiri D, et al. Epileptogenic zone localization using magnetoencephalography predicts seizure freedom in epilepsy surgery. Epilepsia. 2015;56(6):949-58.

43. Gadad V, Sinha S, Mariyappa N, Chaithanya G, Jayabal V, Saini J, et al. Source localization of epileptiform discharges in juvenile myoclonic epilepsy (JME) using magnetoencephalography (MEG). Epilepsy Res. 2017;129:67-73.

44. Murakami H, Wang Zl, Marashly A, Krishnan B, Prayson RA, Kakisaka Y, et al. Correlating magnetoencephalography to stereo-electroencephalography in patients undergoing epilepsy surgery. Brain. 2016;139(11):2935-47.

45. Schmid E, Thomschewski A, Taylor A, Zimmermann G. Diagnostic accuracy of functional magnetic resonance imaging, Wada test, magnetoencephalography, and functional transcranial Doppler sonography for memory and language outcome after epilepsy surgery: A systematic review. Epilepsia 2018;59(12):2305-17.

46. Anninos PA, Tsagas N, Sandyk R, Derpapas K. Magnetic stimulation in the treatment of partial seizures. Int J Neurosci. 1991;60(3-4):141-71.

47. Shon YM, Lim SC, Lim SH. Therapeutic effect of repetitive transcranial magnetic stimulation on non-lesional focal refractory epilepsy. J Clin Neurosci. 2019;63:130-3.

48. Gersner R, Oberman L, Sanchez MJ, Chiriboga N, Kaye HL, Pascual-Leone A, et al. H-coil repetitive transcranial magnetic stimulation for treatment of temporal lobe epilepsy: a case report. Epilepsy Behav Case Rep. 2016;5:52-6.

49. Sun W, Mao W, Meng X, Wang D, Qiao L, Tao W, et al. Low-frequency repetitive transcranial magnetic stimulation for the treatment of refractory partial epilepsy: a controlled clinical study. Epilepsia. 2012;53(10):1782-9.

50. Sun W, Fu W, Mao W, Wang D, Wang Y. Low-frequency repetitive transcranial magnetic stimulation for the treatment of refractory partial epilepsy. Clin EEG Neurosci. 2011:42(1):40-4.

51. Santiago-Rodriguez E, Cardenas-Morales L, Harmony T, Fernandez-Bouzas A, Porras-Kattz E, Hernandez A. Repetitive transcranial magnetic stimulation decreases the number of seizures in patients with focal neocortical epilepsy. Seizure. 2008;17(8):677-83.

52. Joo EY, Han SJ, Chung SH, Cho JW, Seo DW, Hong SB. Antiepileptic effects of low-frequency repetitive transcranial magnetic stimulation by different stimulation durations and locations. Clin Neurophysiol. 2007;118(3):702-8.

53. Fregni $F$, Otachi PT, Do Valle A, Boggio PS, Thut G, Rigonatti SP, et al. A randomized clinical trial of repetitive transcranial magnetic stimulation in patients with refractory epilepsy. Ann Neurol. 2006;60(4):447-55.
54. Kinoshita M, Ikeda A, Begum T, Yamamoto J, Hitomi T, Shibasaki H. Lowfrequency repetitive transcranial magnetic stimulation for seizure suppression in patients with extratemporal lobe epilepsy-a pilot study. Seizure. 2005;14(6):387-92.

55. Fregni F, Thome-Souza S, Bermpohl F, Marcolin MA, Herzog A, PascualLeone A, et al. Antiepileptic effects of repetitive transcranial magnetic stimulation in patients with cortical malformations: an EEG and clinical study. Stereotact Funct Neurosurg. 2005;83(2-3):57-62.

56. Menkes DL, Gruenthal M. Slow-frequency repetitive transcranial magnetic stimulation in a patient with focal cortical dysplasia. Epilepsia. 2000;41(2):240-2.

57. Cooper YA, Pianka ST, Alotaibi NM, Babayan D, Salavati B, Weil AG, et al. Repetitive transcranial magnetic stimulation for the treatment of drugresistant epilepsy: a systematic review and individual participant data metaanalysis of real-world evidence. Epilepsia Open. 2018;3(1):55-65.

58. Dong $L$, Zheng $Y, L i Z Y$, Li G, Lin L. Modulating effects of on-line low frequency electromagnetic fields on hippocampal long-term potentiation in young male Spraque-Dawley rat. J Neurosci Res 2018;96(11):1775-85.

59. Zheng Y, Ma W, Dong L, Dou JR, Gao Y, Xue J. Influence of the on-line ELFEMF stimulation on the electrophysiological properties of the rat hippocampal CA1 neurons in vitro. Rev Sci Instrum. 2017;88(10):105106.

60. Ghai RS, Bikson M, Durand DM. Effects of applied electric fields on lowcalcium epileptiform activity in the CA1 region of rat hippocampal slices. J Neurophysiol. 2000;84(1):274-80.

61. Ye H, Kaszuba S. Neuromodulation with electromagnetic stimulation for seizure suppression: from electrode to magnetic coil. IBRO Rep. 2019;7:26-33.

\section{Ready to submit your research? Choose BMC and benefit from:}

- fast, convenient online submission

- thorough peer review by experienced researchers in your field

- rapid publication on acceptance

- support for research data, including large and complex data types

- gold Open Access which fosters wider collaboration and increased citations

- maximum visibility for your research: over $100 \mathrm{M}$ website views per year

At BMC, research is always in progress.

Learn more biomedcentral.com/submissions 